\title{
Pharmacogenomics: where does Britain stand?
}

"...the UK, and indeed the rest of the EU needs to copy the Scandinavians in educating the public. This is a responsibility incumbent on academics, practitioners and those in government."

\section{KEYWORDS: biobanks " pharmacogenomics - public attitudes $\approx$ regulation}

Pharmacogenetics and pharmacogenomics offer much potential for better targeted treatment, delivering better care more efficiently to the advantage of patients, government and industry. However, its development is being held back by regulatory overlap in the EU and thus the UK. At a time of almost unprecedented economic crisis, this is a luxury we cannot afford. However, there is considerable heterogeneity of views amongst the public of different EU countries, which may make harmonization difficult. There is also widespread public ignorance of biobanks in particular, and pharmacogenomics in general. In this respect the UK, and indeed the rest of the EU needs to copy the Scandinavians in educating the public. This is a responsibility incumbent on academics, practitioners and those in government.

Pharmacogenetics and pharmacogenomics are often used and discussed together and we use the term PGx to collectively refer to both technologies. PGx provides opportunities for better, more targeted treatment, treatment which reduces the incidence of adverse reactions and increases that of positive ones. It provides opportunities for pharmaceutical firms to develop successful products more efficiently [101]. It also has the potential to enhance pharmacovigilance [1]. Thus, it potentially offers benefits to patients, hospitals, insurance firms and state funders of healthcare. In helping pharmaceutical firms, it offers a potential advantage to the European and UK industry, and at a time of almost unprecedented economic crisis, that is important. However, it is also clear that the full benefits of PGx are a long way from being realized $[2,3]$.

Biobanks play a key role in PGx. These are banks of data on biological material (e.g., tissue and blood) combined with donor-related clinical information. They facilitate drug discovery and the identification and validation of biomarkers by delivering data on need. As an example, the
UK biobank is collecting data on 500,000 individuals aged between 40 and 69 years of age [102]. Attempts are being made to create a European network of such data, although this is hampered by the heterogeneity of the different databases. A further, and rather different problem is data privacy. This is part of a larger problem where a growing amount of personal individual information is stored electronically. If this were to be brought together by government or private agents, it would pose a significant danger to individual liberties.

There are concerns that PGx could lead to the stratification of populations based on genetic variants, with some population groups proving too small to be of interest to the pharmaceutical industry. PGx may also impact on insurance firms' behavior as well as employment opportunities. Most European countries have introduced legislation aimed against genetic discrimination, although it remains to be seen how effective this will eventually prove to be [3]. There are also concerns that initially new innovations will be expensive and only gradually filter through to the entire population. Finally, new technologies often require expertise and mistakes are possible [4].

\section{Regulation}

PGx raises specific regulatory problems. Drugs and diagnostics often need to be jointly regulated. In the EU, in contrast to the USA, the licensing of therapeutics and diagnostic products is done separately, and applications to market the latter must be made to the national agencies. The in vitro diagnostic directive is central to this [5]. A European Conformity Mark is required (i.e., a manufacture's declaration that the product complies to all relevant legislation) [6]. Despite this, there are substantial differences in the regulation of in vitro diagnostics between member states [7]. European Commission directives also govern biobanking. The EU is trying to harmonize the

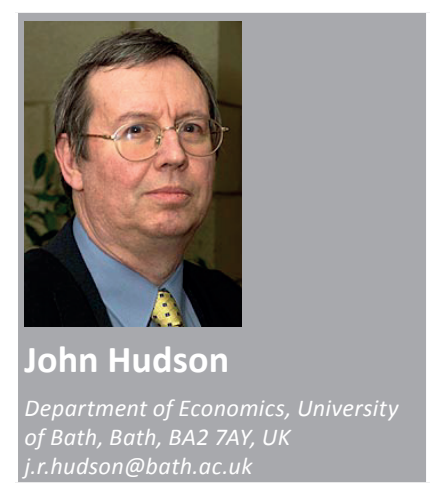

Future $\mathrm{fSS}$ 
system [8]; however at the moment, member states interpret the directives in different ways leading to substantially different national systems. The system of split responsibility has the potential for regulatory delay and even disagreement and conflict.

New products have to be regulated and controls put in place to ensure that they are reasonably safe. It is difficult, if not impossible, to ensure absolute safety; however, such regulation often slows down the innovation process [9], adding to firm's costs and perhaps disadvantaging EU firms [10]. Thus, for example, most European patents take more than 60 months to process, whereas most US patents are processed in 30-60 months [11]; however, the balancing act between encouraging innovation and protecting public safety is a fine one to achieve, and it is not immediately obvious as to whether the USA or the EU has this right. The Clinical Trials Directive, which is currently under review, has faced particular criticism that despite some benefits, it has led to a significant decline in the attractiveness of EU-based research [103].

\section{"The level of ignorance on biobanks is shocking. In the UK, 67\%, and in France 75\%, of people asked had not heard of biobanks..."}

The role of patents causes particular problems. Paci et al. conclude that although there is some evidence to suggest that patents and the European patent system is causing a problem to PGx research and development, it is not yet definitive enough to draw firm conclusions [12]. One of the problems in this area is that it is relatively hard to detect the incidence and causes of something that does not happen; for example, no new product innovations, compared with events that do happen. Patents can both encourage innovation by protecting intellectual property rights, and discourage it, by making the process of innovation more complex and expensive. As technology becomes more complex, multiplatformed and interconnected, the patent system is increasingly under the microscope and not simply with respect to pharmaceutical products.

\section{Public attitudes to PGx}

We now compare attitudes to different aspects of PGx in the UK with those in the EU. The data, which dates from 2010, relates to a specific aspect of PGx, namely biobanks. The data come from Eurobarometer 73.1, a survey of European public opinion carried out in early 2010 which saw approximately 1000 people surveyed in each EU country. There are wide differences on attitudes to biobank cooperation across the EU. The Scandinavian countries see substantial willingness to cooperate - this is slightly less in the UK than in the EU as a whole, but roughly on a par with France. In terms of socioeconomic characteristics, willingness to cooperate is greater for men and increases with the level of education. The data suggested that being a Muslim or unemployed are factors that are associated with unwillingness to cooperate. The results with respect to education and Muslims carry over to other EU countries, but less so to those relating to the unemployed and gender.

When we perform regression analysis (using the technique of ordered probit) on these attitudes, we find that for the EU, willingness to cooperate is less in regions, within countries, with high unemployment, for those living in villages and increases with age and levels of education. To an extent this is consistent with people being influenced by self-interest, as those in rural and poorer areas are least likely to benefit from technical advances such as PGx [5]. It is also greater for those who profess no religion and least for Muslims, which may indicate the influence of culture. It suggests take up will be lower for Muslims, who in the UK are primarily Pakistani, but in Germany are Turkish and in France, north African. This may then have repercussions on targeted drug development for these ethnic groups. However, it may not be being Islamic, but nonChristian which is relevant; there were very few Jewish or Hindu individuals in the sample. This deserves further analysis.

When asked about their specific concerns, of those who expressed an opinion, over $36 \%$ in the UK said none, compared with $28.3 \%$ in the EU as a whole. In the UK, most concern was expressed with respect to providing medical records, closely followed by genetic profiles. The provision of operational tissue, blood and lifestyle evoked slightly less concern. In all of these aspects, the Swedes stood out as being amongst the least concerned and the Germans among the most.

The level of ignorance on biobanks is shocking. In the UK, 67\%, and in France 75\%, of people asked had not heard of biobanks; however, this was more than the EU average of $60 \%$. In Sweden it was just $22 \%$. Knowledge increased with education, but only slightly. At the other end of the scale, for the unemployed the figure was $80 \%$ and for Muslims it was $82 \%$. In terms of who should be primarily responsible for protecting the public interest, $46 \%$ of UK respondents placed the emphasis on doctors, followed by national governments (36\%) and national data-protection 
agencies (28\%); however there were substantial differences between the different EU countries. Further details on all these results are available from the author on request.

In many respects, an earlier survey, carried out in 2005 (Eurobarometer survey 64.3 carried out in November/December 2005. The individual responses only become available in 2009), showed similar results, particularly with respect to socioeconomic characteristics. This looked more generally at PGx with respect to the EU as a whole, and has been discussed by Hudson and Orviska [5]. It too highlighted awareness of PGx to be particularly low in the UK, with only $14.2 \%$ having heard of it, compared with Sweden where the figure was $49.4 \%$.

\section{Conclusion}

To answer the question posed in the title, Britain stands in a state of some confusion and ignorance. The country, as indeed the EU as a whole, faces many challenges with respect to the economic situation, the pressure on public-sector spending and the aging population. In these respects, technology can help, and help substantially, in providing better and more efficient healthcare and in enhancing the competitiveness of UK pharmaceutical companies (which represent one of the relatively few largely unqualified UK success stories as we move forward). There are, however, a number of obstacles which stand in the way of its development.

Many of these issues are specific to the UK and the EU. A lack of cohesion and a diversity of regulations and authorities is hampering research and development in PGx [101]. If the EU is to continue in its present form, and this is a probability, but by no means a certainty, then it simply has to move towards a more federal, harmonized structure in this and other respects. With respect to PGx, this will not be easy given the heterogeneity of views noted above and, of course, will also present particular political problems in the UK.

New innovations need public support, as without this, diffusion will be slow. From an ethical point of view too, it is right that the issues, often complex, are explained to society rather than foisted on it behind a veil of ignorance. In the EU, this is hampered by widespread ignorance of concepts such as PGx and biobanks, and this ignorance is particularly great in the UK. In strong contrast to this, in Sweden there is much less ignorance of biotechnology and more support. Put simply, we need to imitate the Swedes more; however, it is arguably not just the public who need educating, but it is also the physicians [1], and certainly in other countries and perhaps the UK too, practicing pharmacists, laboratory staff and nurses [13].

\section{Financial \& competing interests disclosure}

The author has no relevant affliations or financial involvement with any organization or entity with a financial interest in or financial conflict with the subject matter or materials discussed in the manuscript. This includes employment, consultancies, honoraria, stock ownership or options, expert testimony, grants or patents received or pending, or royalties.

No writing assistance was utilized in the production of this manuscript.

\section{References}

1 Shih T, Vourvahis M, Singh M, Papay J. Pharmacogenetics: from bench science to the bedside. Drug Inf. J. 42(5), 503-513 (2008).

2 Zaza G, Granata S, Sallustio F, Grandaliano G, Schena FP. Pharmacogenomics: a new paradigm to personalize treatments in nephrology patients. Clin. Exp. Immunol. 159(3), 268-280 (2010).

3 Vijverberg SJH, Pieters T, Cornel MC. Ethical and social issues in pharmacogenomics testing. Curr. Pharm. Des. 16(2), 245-252 (2010).

4 Ibarreta D, Elles R, Cassiman JJ et al. Towards quality assurance and harmonization of genetic testing services in the European Union. Nature Biotechnol. 22(10), 1230-1235 (2004).

5 Hudson J, Orviska M. European attitudes to gene therapy and pharmacogenetics. Drug Discov. Today 16(19-20), 843-847 (2011).

6 Dati F, Denoyal G, van Helden J. European performance evaluations of the ADVIA Centaur infectious disease assays: requirements for performance evaluation according to the European directive on in vitro diagnostics. J. Clin. Virol. 30(1), S6-S10 (2004).

7 Kuzma J, Najmaie P, Larson J. Evaluating oversight systems for emerging technologies: a case study of genetically engineered organisms. J. Law Med. Ethics 37(4), 546-586 (2009).

8 Gaisser S, Vignola-Gagne E, Husing B et al. EU policies in personalized medicine-related technologies. Pers. Med. 6(1), 93-102 (2007).

9 Salerno RA, Lesko LJ. Pharmacogenomics in drug development and regulatory decision making: The Genomic Data Submission (GDS) Proposal. Pharmacogenomics 5(1), 25-30 (2004).

10 Gurwitz D, Zika E, Hopkins MM et al. Pharmacogenetics in Europe: barriers and opportunities. Public Health Genomics 12(3), 134-141 (2009).

11 Hopkins MM, Mahdi S, Patel P et al. DNA patenting: the end of an era? Nature Biotechnol. 25(2), 185-187 (2007).
12 Paci D, Hopkins MM, Zika E et al. Impact of DNA patents on pharmacogenomics research and development. Economic and Policy Issues 71(8), 485-491 (2010).

13 Chen HY, Chen LC. Implementation of innovative pharmacogenetic tests into practice in Taiwan: an institutional perspective. Drug Dev. Res. 71(8), 502-506 (2010).

\section{Websites}

101 UK Pharmacogenetics Study Group. Policy Issues in Pharmacogenetics. University of York, York, UK (2006). www.york.ac.uk/media/satsu/res-pgx/ PGxpolicyissues2006.pdf

102 UK BioBank. www.ukbiobank.ac.uk

103 European Commission. Assessment of the functioning of the 'Clinical Trials Directive' 2001/20/EC: Public consultation paper (2009). http://ec.europa.eu/health/files/clinicaltrials/ docs/2009_10_09_public-consultationpaper.pdf 